\title{
A simple strategy to improve the yield of graphene nanosheets in the anodic exfoliation of graphite foil
}

\author{
J.M. Munuera*, J.I. Paredes, S. Villar-Rodil, A. Martínez-Alonso, J.M.D. Tascón \\ Instituto Nacional del Carbón, INCAR-CSIC, Apartado 73, 33080 Oviedo, Spain
}

\begin{abstract}
Strategies that improve the yield of graphene nanosheets in anodic exfoliation processes are desirable for the mass production of this material. Here, a simple pretreatment of graphite foil with concentrated $\mathrm{H}_{2} \mathrm{SO}_{4}$ is demonstrated to be such an effective strategy, affording overall exfoliation yields up to $\sim 50 \mathrm{wt} \%$ under certain experimental conditions, compared with a baseline value of $\sim 10 \mathrm{wt} \%$ for untreated graphite. Filling of inner voids and interstices of the graphite foil with $\mathrm{H}_{2} \mathrm{SO}_{4}$ molecules was concluded to be responsible for the enhanced exfoliation performance. Additional approaches to further increase the anodic exfoliation yield are also proposed and discussed.
\end{abstract}

At present, the prospects for the large-scale production of graphene face several hurdles that stand in the path of the widespread practical implementation of this twodimensional carbon material. Bottom-up production methods (most notably, chemical vapor deposition) can afford monolayer graphene wafers of a high structural quality and large lateral dimensions, but the conditions required for their synthesis are generally not amenable for massive up-scaling [1]. On the other hand, mainstream approaches based on exfoliation of graphite and graphite derivatives (top-down methods) suffer either from a very low crystalline quality of the exfoliated material (e.g., the graphite oxide route) or from low yield ( $<5 \mathrm{wt} \%)$ and incomplete exfoliation (typical flake thickness >3-4 monolayers) of the starting graphites (e.g., direct liquid-phase exfoliation routes reliant on sonication or shear forces) [1,2].

\footnotetext{
${ }^{*}$ Corresponding author. Phone number: $(+34) 985119090$. E-mail address: j.munuera@incar.csic.es (Jose Munuera)
} 
In this context, electrochemical exfoliation methods, which rest on ion intercalation of a graphite electrode triggered by an applied DC bias voltage, have recently emerged as a promising alternative $[3,4,5,6,7,8]$. With the use of proper electrolytes and solvent media, both cathodic and anodic exfoliation is possible, the latter being particularly attractive and more usually deployed because it can be carried out in aqueous solutions of common electrolytes, such as $\mathrm{H}_{2} \mathrm{SO}_{4}$ or its inorganic salts [4]. Concerning the electrode material for exfoliation, graphite foil is a frequent choice owing to its modest cost $(\sim 10-20$ per kilogram $)$ as well as morphological features that are especially conducive to facilitating delamination [9]. However, although graphenes produced by anodic exfoliation tend to possess a higher structural quality than that of their graphite oxide-derived counterparts and are better exfoliated than the typical nanosheets obtained via direct sonication/shear routes, in many cases the overall yield of exfoliation is rather low (e.g., $~ 10 \mathrm{wt} \%$ for graphite foil) [10], with the effect of different process parameters on the yield being seldom investigated [4]. Thus, the development of rational strategies aimed at increasing this yield is needed if anodic exfoliation is to become a truly competitive option for the mass production of graphene. We report here that a simple pre-treatment of graphite foil with concentrated $\mathrm{H}_{2} \mathrm{SO}_{4}$ is one such effective strategy, as it affords much improved exfoliation yields during the subsequent anodic treatment in aqueous electrolyte. $\mathrm{H}_{2} \mathrm{SO}_{4}$ is a common and widely used reactive, particularly in the preparation of graphene oxide via the Hummers method and in the anodic exfoliation of graphite into graphene. We also discuss the possible mechanisms responsible for the observed behavior as well as some approaches to further increase the yield.

Treatment of graphite foil pieces $\sim 0.5 \mathrm{~mm}$ in thickness with concentrated $\mathrm{H}_{2} \mathrm{SO}_{4}$ at room temperature for a given period of time [see Electronic Supplementary Material (ESM) for a description of the experimental procedure] afforded highly hygroscopic materials, as visually evidenced by the condensation of water droplets on their surface upon exposure to ambient air. Field emission scanning electron microscopy (FE-SEM) imaging indicated that the graphite foil morphology, which was originally comprised of random stacks of micrometer-sized graphite platelets and exhibited a high density of folds, wrinkles and overlaps with voids and interstitial spaces in-between (Fig. 1a), became slightly rougher and developed a number of dome-like features (blisters) after exposure to $\mathrm{H}_{2} \mathrm{SO}_{4}$ (Fig. 1b). The latter were ascribed to $\mathrm{H}_{2} \mathrm{SO}_{4}$ molecules trapped in the voids/interstices of the material. Moreover, prolonged exposure of the graphite foil surface to the electron beam from the FE-SEM apparatus led to the progressive 
disappearance of the blisters (images not shown), probably as a result of outward diffusion of the $\mathrm{H}_{2} \mathrm{SO}_{4}$ molecules induced by a local temperature rise.
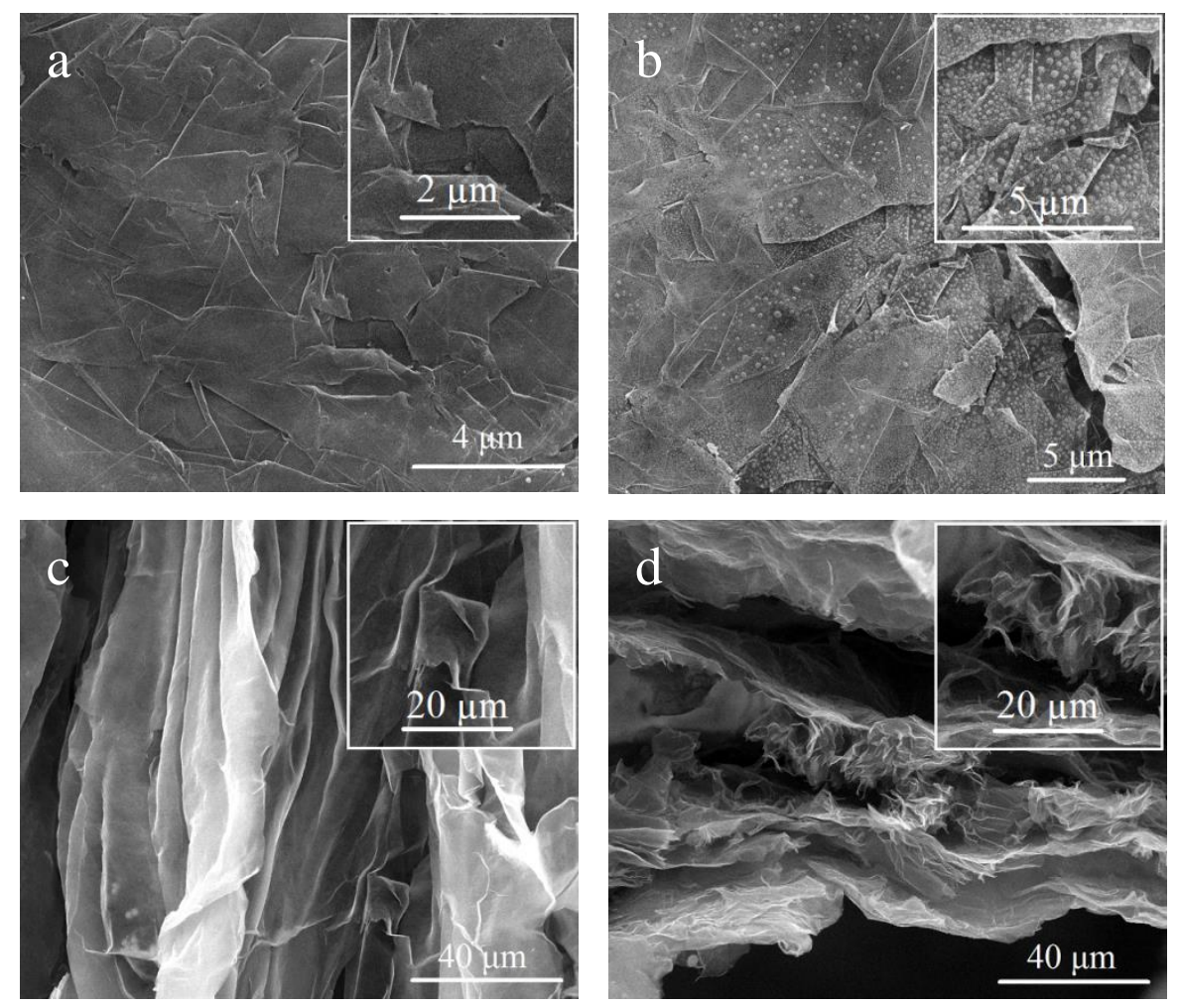

Figure 1. (a,b) Typical FE-SEM images of the surface of (a) untreated and (b) $\mathrm{H}_{2} \mathrm{SO}_{4}$ treated (6 h) graphite foil. (c,d) FE-SEM images of anodically expanded graphite foil (c) without and (d) with $\mathrm{H}_{2} \mathrm{SO}_{4}$ pre-treatment $(6 \mathrm{~h})$.

The graphite foil samples (both untreated and treated with $\mathrm{H}_{2} \mathrm{SO}_{4}$ for different periods of time) were then subjected to anodic exfoliation in aqueous $0.1 \mathrm{M} \mathrm{K}_{2} \mathrm{SO}_{4}$ solution (see ESM for details). In all cases, a significant fraction of the graphite material was seen to expand and detach from the anode, as expected. FE-SEM inspection of the expanded products after extensive washing with deionized water (Fig. 1c and d) provided microscopic evidence of their delamination, revealing very thin, corrugated layers that were separated by submicrometer-/ micrometer-sized voids. Such a type of morphology is characteristic of successful anodic exfoliation processes [10,11]. No significant morphological differences between the untreated (Fig. 1c) and any of the $\mathrm{H}_{2} \mathrm{SO}_{4}$-treated graphite foil specimens (e.g., Fig. 1d) were apparent after the anodic expansion. However, the net amount of expanded product that was generated from the pre-treated samples was usually much larger than that obtained from their untreated 
counterpart, thus providing a first hint of the beneficial effect of such a pre-treatment on exfoliation.

To provide a quantitative measure of the overall yield of exfoliation, the anodically expanded products were bath-sonicated in $N, N$-dimethylformamide (DMF), water/isopropanol mixture $(65 / 35 \mathrm{v} / \mathrm{v} \%)$ or in aqueous solution of flavin mononucleotide (FMN) [9], and then centrifuged to separate the individual, wellexfoliated graphene nanosheets (supernatant) from the incompletely delaminated material (sediment). The exfoliation yield was calculated as the ratio of the amount of graphene nanosheets dispersed in the solvent (estimated by means of UV-vis absorption spectroscopy [10]) to the original mass of the graphite foil electrode. The results obtained for different treatment times of the graphite foil with $\mathrm{H}_{2} \mathrm{SO}_{4}$ are presented in Fig. 2, where it is apparent that such an approach had a sizable positive impact on the exfoliation yield. Specifically, the yield increased from a baseline value of $\sim 10 \mathrm{wt} \%$ attained with untreated graphite foil under the experimental conditions described in the ESM up to $\sim 50 \mathrm{wt} \%$ for treatment times longer than $\sim 40 \mathrm{~h}$, i.e., a 5-fold increase in yield was accomplished. However, short treatment times already afforded much enhanced yields (e.g., a $\sim 3$-fold increase was observed after $5 \mathrm{~h}$ ). We note that these yield values exhibited a variance of $\sim 10-20 \%$ for different samples prepared under exactly the same conditions, as it is sometimes observed for electrochemically exfoliated graphene. It is important to note that the criterion followed here to determine the exfoliation yield was more stringent than others adopted in the literature. For instance, in some cases the yield has been calculated as the amount of anodically expanded product (before dispersion in any solvent) relative to the starting mass of the graphite anode [11], or as the amount of solvent-dispersed graphene relative to the mass of anodically expanded product (rather than the total mass of the graphite anode) [12]. Likewise, certain process parameters must have a critical effect on the exfoliation yield (e.g., thinner graphite foils are expected to afford higher yields), but in many studies such parameters are not provided. For these reasons, meaningful comparisons with previous yield data from the literature are difficult to make, as such data cannot be taken in absolute terms. Nevertheless, the present results clearly demonstrate that large increases in the anodic exfoliation yield (and thus in the amount of generated graphene per unit time) can be attained just by dipping the graphite foil in concentrated $\mathrm{H}_{2} \mathrm{SO}_{4}$. 


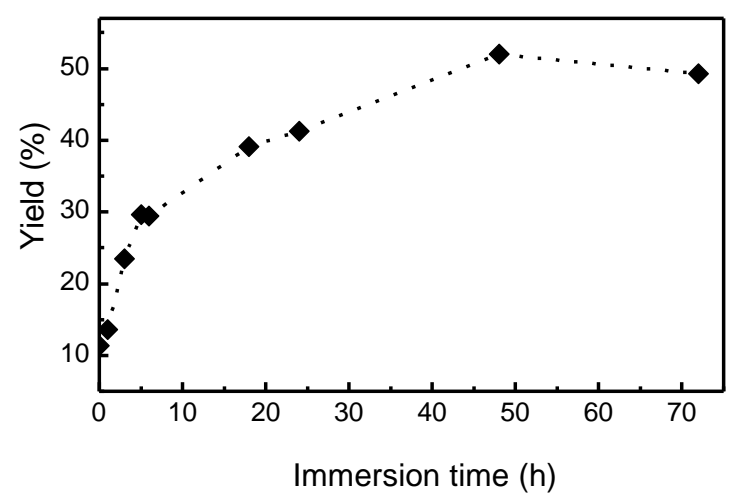

Figure 2. Anodic exfoliation yield of graphite foil as a function of $\mathrm{H}_{2} \mathrm{SO}_{4}$ pre-treatment time. The yield was determined as the amount of solvent-dispersed graphene nanosheets obtained relative to the initial mass of the graphite foil anode.

Physicochemical characterization of the anodically delaminated products confirmed the generation of graphene nanosheets from $\mathrm{H}_{2} \mathrm{SO}_{4}$-treated graphite foil. Fig. 3a shows the a representative UV-vis absorption spectrum of a colloidal dispersion obtained in water/isopropanol mixture from graphite pre-treated in $\mathrm{H}_{2} \mathrm{SO}_{4}$ for $24 \mathrm{~h}$ (see inset for a digital picture of the dispersion), where a peak at $\sim 268 \mathrm{~nm}$ and strong absorbance in the 270-1000 nm range revealed the presence of suspended graphitic material [9]. This spectrum was characteristic of those obtained for graphene dispersions obtained from graphite foil electrodes dipped in $\mathrm{H}_{2} \mathrm{SO}_{4}$ for different times. Atomic force microscopy (AFM) imaging showed that this material was made up of nanosheets typically between a few and several hundred nanometers in lateral size with an apparent thickness of $\sim 1$ $1.5 \mathrm{~nm}$ (Fig. 3b), consistent with few-layer (<4) graphene. As revealed by X-ray photoelectron spectroscopy (XPS), pre-treatment with $\mathrm{H}_{2} \mathrm{SO}_{4}$ led to anodically exfoliated nanosheets having an increased extent of oxidation (Fig. 3c). Indeed, the O/C atomic ratio for graphenes obtained from foils pre-treated for long times was $\sim 0.20$, compared with a value of $\sim 0.10$ for graphenes derived from untreated graphite foil. However, such a level of oxidation was well within the range of values typically reported for graphenes prepared by anodic exfoliation in $\mathrm{H}_{2} \mathrm{SO}_{4}$ or sulfate salt electrolytes (O/C ratios 0.10-0.30) [10,13,14]. Moreover, Raman spectroscopy indicated that the structural quality of the graphene nanosheets was not seriously degraded as a result of pre-treatment of the graphite foil with $\mathrm{H}_{2} \mathrm{SO}_{4}$, since no significant changes to the D/G band ratios were observed (Fig. 3d). For comparison, the spectrum of hydrazine-reduced graphene oxide exhibited broader D and G Raman bands 
[FWHM(G band) $\sim 80 \mathrm{~cm}^{-1}$ vs. $\sim 50 \mathrm{~cm}^{-1}$, respectively, see inset to Fig. 3d), thus denoting a more disordered carbon lattice. Conductivity measurements were carried out for the samples prepared with a $24 \mathrm{~h}$ dipping in $\mathrm{H}_{2} \mathrm{SO}_{4}$, yielding a value of $\sim 35000 \mathrm{~S} \mathrm{~m}^{-1}$. This value is in the range of conductivities typically measured for good quality electrochemically exfoliated graphene flakes $[9,10]$, suggesting that the structural quality of the flakes has not been diminished to a great extent because of the pretreatment, in agreement with the Raman data.
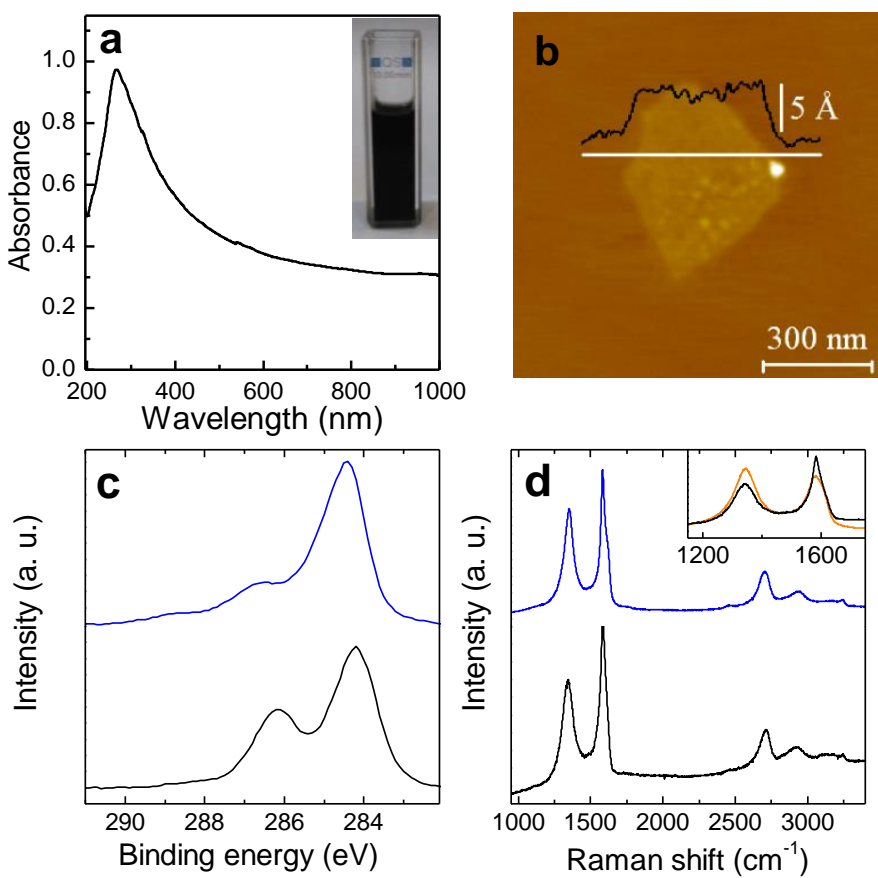

Figure 3. (a) UV-vis absorption spectrum of a colloidal dispersion of anodically exfoliated graphene in water/isopropanol mixture $(65 / 35 \mathrm{v} / \mathrm{v} \%)$ obtained from a $\mathrm{H}_{2} \mathrm{SO}_{4^{-}}$ treated graphite foil. Inset: digital picture of the dispersion. (b) AFM image of graphene nanosheets anodically exfoliated from graphite foil pre-treated with $\mathrm{H}_{2} \mathrm{SO}_{4}(24 \mathrm{~h})$. A representative line profile (green trace) taken along the marked white line is shown overlaid on the image. (c) High resolution C 1s core level XPS and (d) Raman spectra of graphene nanosheets anodically exfoliated from (i) untreated and (ii) $\mathrm{H}_{2} \mathrm{SO}_{4}$-treated (24 h) graphite foil. The inset to (d) compares the first-order Raman spectrum of the latter sample (black trace) with that of hydrazine-reduced graphene oxide (orange trace).

To understand the origin of the positive effect of $\mathrm{H}_{2} \mathrm{SO}_{4}$ pre-treatment on the anodic exfoliation yield of graphite foil, we first note that insufficient intercalation of the graphite anode during the electrolytic process was probably responsible for the limited 
exfoliation yield observed with the untreated material. Hence, approaches that boost the extent of ion intercalation should be expected to give rise to increased yields. Second, as noted above, graphite foil incorporates a large number of voids and interstitial spaces in its structure (the density of the material used here was $0.7-1.3 \mathrm{~g} \mathrm{~cm}^{-3} \mathrm{vs} \sim 2.26 \mathrm{~g} \mathrm{~cm}^{-3}$ for defect-free, single-crystal graphite [15]). These voids and interstices can be assumed to become progressively filled with $\mathrm{H}_{2} \mathrm{SO}_{4}$ molecules during exposure to the concentrated acid, thus making the material hygroscopic. When such a pre-filled graphite is subjected to anodic treatment in aqueous electrolyte, the very high local density of $\mathrm{SO}_{4}{ }^{2-}$ anions inside the graphite foil should promote an extensive initial intercalation of the material that would facilitate the further entrance of anions from the aqueous medium, which in turn would result in a more extensive delamination of the anode. We believe void filling to be the only effect of $\mathrm{H}_{2} \mathrm{SO}_{4}$ pre-treatment, incorporation of the acid in the graphite interlayer spaces to give $\mathrm{H}_{2} \mathrm{SO}_{4}$-graphite intercalation compounds (GICs) being reasonably ruled out: X-ray diffraction did not support the formation of such GICs (Fig. S1 in the ESM), which generally requires the presence of an oxidizing species in addition to $\mathrm{H}_{2} \mathrm{SO}_{4}$ [16]. The key role played by the high density of voids/interstices was made apparent in a control experiment in which graphite foil was replaced by a voidfree graphite, namely, highly oriented pyrolytic graphite (HOPG; density $\sim 2.25 \mathrm{~g} \mathrm{~cm}^{-3}$ ). In this case, pre-treatment of $\mathrm{HOPG}$ with $\mathrm{H}_{2} \mathrm{SO}_{4}$ did not lead to a large increase in the exfoliation yield. A schematic illustration of the proposed effect of $\mathrm{H}_{2} \mathrm{SO}_{4}$ pre-treatment on the anodic exfoliation of graphite foil is provided in Fig. 4.

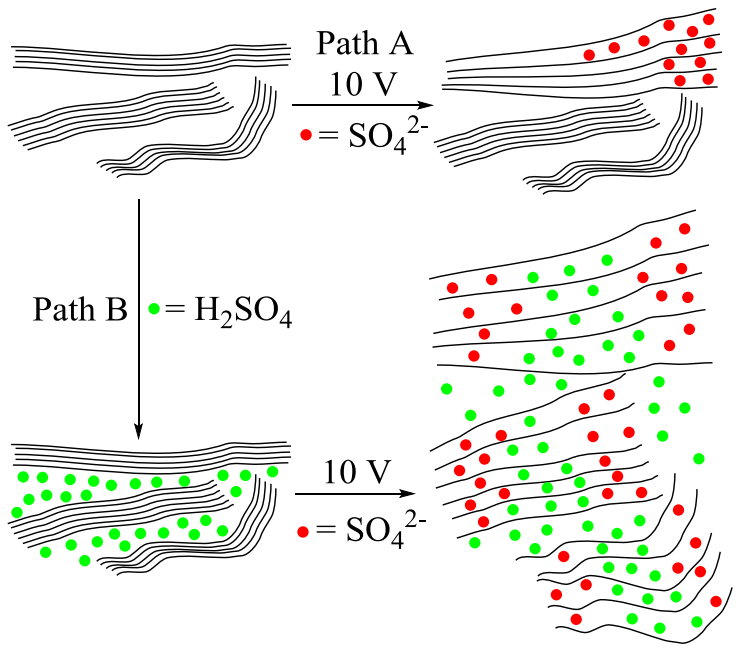

Figure 4. Schematic of the proposed anodic exfoliation process of graphite foil without and with $\mathrm{H}_{2} \mathrm{SO}_{4}$ pre-treatment. When no pre-treatment is undertaken (path $\mathrm{A}$ ), limited access of the aqueous anions from the electrolyte (orange circles) to the interior of the 
foil leads to a low exfoliation yield. When a $\mathrm{H}_{2} \mathrm{SO}_{4}$ pre-treatment is carried out (path B), the acid molecules filling the inner voids and interstices of the graphite foil (red circles, step B1) promote a more extensive intercalation of the material during the subsequent anodic process, resulting in a higher exfoliation yield (step B2).

We note that simply increasing the aqueous electrolyte concentration as an alternative to the $\mathrm{H}_{2} \mathrm{SO}_{4}$ pre-treatment is not an effective strategy, as it does not usually lead to higher exfoliation yields [11]. In aqueous medium, access of the electrolyte anions to the inner voids and interstices of the foil is probably restricted by the hydrophobic nature of the material. Such a restriction should be lifted when these voids/interstices become hygroscopic as a result of their filling with $\mathrm{H}_{2} \mathrm{SO}_{4}$, an effect that would also contribute to increasing the exfoliation yield. On the other hand, dipping the graphite foil in liquid $\mathrm{N}_{2}$ followed by soaking in ethanol, which created macroscopic cracks and voids in the material, led to a $\sim 2$-fold increase in anodic exfoliation yield (up to $\sim 20 \mathrm{wt} \%$ ). However, combined liquid $\mathrm{N}_{2} / \mathrm{H}_{2} \mathrm{SO}_{4}$ treatment did not increase the overall exfoliation yield significantly above $50 \mathrm{wt} \%$. Working with lower density versions of graphite foil (densities as low as $\sim 0.05 \mathrm{~g} \mathrm{~cm}^{-3}$ have been produced [17]) should be a better option than the aggressive liquid $\mathrm{N}_{2}$ treatment, as the higher numbers of small-sized voids and interstices should favor an increased and more uniform filling of the material with $\mathrm{H}_{2} \mathrm{SO}_{4}$ molecules that would result in higher exfoliation yields. Other process parameters should also bear an influence on the exfoliation yield (e.g., we have observed that higher electrolyte volume/graphite anode mass ratios as well as thinner foils lead to higher yields), but their effect has not yet been systematically investigated, which should warrant further studies along these lines in the future.

In summary, we have shown that pre-treatment with concentrated $\mathrm{H}_{2} \mathrm{SO}_{4}$ is an effective strategy to increase the anodic exfoliation yield of graphite foil in aqueous electrolyte. Such an increase was thought to arise from a more extensive intercalation of this material during the electrolytic process due to the filling of its voids/interstices with $\mathrm{H}_{2} \mathrm{SO}_{4}$ molecules. This simple approach should contribute to making anodic exfoliation a more competitive method for the large-scale production of graphene.

\section{Acknowledgements}

Financial support from the Spanish Ministerio de Economía y Competitividad (MINECO) and the European Regional Development Fund (ERDF) through project 
MAT2015-69844-R is gratefully acknowledged. Partial funding by Plan de Ciencia, Tecnología e Innovación 2013-2017 del Principado de Asturias and the ERDF (project GRUPIN14-056) is also acknowledged. J.M.M. is grateful to the Spanish Ministerio de Educación, Cultura y Deporte (MECD) for his pre-doctoral contract.

\section{References}

[1] A.C. Ferrari, F. Bonaccorso, V.I. Fal'ko, K.S. Novoselov, S. Roche, P.Bøggild, et al., Science and technology roadmap for graphene, related two-dimensional crystals, and hybrid systems, Nanoscale 7 (2015), 4598-810.

[2] A. M. Dimiev, G. Ceriotti, A. Metzger, N. D. Kim, J.M. Tour, Chemical mass production of graphene nanoplatelets in $~ 100 \%$ yield, ACS Nano 10, (2016), 274-9

[3] A.M. Abdelkader, A.J. Cooper, R.A.W. Dryfe, I.A. Kinloch, How to get between the sheets: a review of recent works on the electrochemical exfoliation of graphene materials from bulk graphite Nanoscale 7 (2015), 6944-56.

[4] A. J. Cooper, N. R. Wilson, I. A. Kinloch, R. A. W. Dryfe, Single stage electrochemical exfoliation method for the production of few-layer graphene via intercalation of tetraalkylammonium cations, Carbon 66, (2014), 340-50

[5] S. Yang, M.R. Lohe, K. Müllen, X. Feng, New-Generation Graphene from Electrochemical Approaches: Production and Applications, Adv. Mater. 28 (2016), 6213-21.

[6] H. J. Salavagione, Promising alternative routes for graphene production and functionalization, J. Mater. Chem. A, 2, (2014), 7138-46

[7] G.M. Morales, P. Schifani, G. Ellis, C. Ballesteros, G. Martínez, C. Barbero et al, High-quality few layer graphene produced by electrochemical intercalation and microwave-assisted expansion of graphite, Carbon 49, (2011), 2809

[8] A. Ambrosi, M. Pumera, Electrochemically exfoliated graphene and graphene oxide for energy storage and electrochemistry applications, Chem. Eur. J, 22, (2016), 153-9

[9] J.M. Munuera, J.I. Paredes, S. Villar-Rodil, M. Ayán-Varela, A. Pagán, S.D. AznarCervantes, et al., High quality, low oxygen content and biocompatible graphene nanosheets obtained by anodic exfoliation of different graphite types, Carbon 94 (2015), 729-39.

[10] J.M. Munuera, J.I. Paredes, S. Villar-Rodil, M. Ayán-Varela, A. Martínez-Alonso, J.M.D. Tascón, Electrolytic exfoliation of graphite in water with multifunctional electrolytes: en route towards high quality, oxide-free graphene flakes, Nanoscale 8 (2016), 2982-98.

[11] K. Parvez, Z.-S. Wu, R. Li, X. Liu, R. Graf, X. Feng, et al., Exfoliation of Graphite into Graphene in Aqueous Solutions of Inorganic Salts, J. Am. Chem. Soc. 136 (2014), 6083-91.

[12] B.D. Ossonon, D. Bélanger, Functionalization of graphene sheets by the diazonium chemistry during electrochemical exfoliation of graphite, Carbon 111 (2017), 83-93.

[13] J. Liu, M. Notarianni, G. Will, V.T. Tiong, H. Wang, N. Motta, Electrochemically Exfoliated Graphene for Electrode Films: Effect of Graphene Flake Thickness on the Sheet Resistance and Capacitive Properties, Langmuir 29 (2013), 13307-13314. 
[14] J. Liu, C.K. Poh, D. Zhan, L. Lai, S.H. Lim, L. Wang, et al, Improved synthesis of graphene flakes from the multiple electrochemical exfoliation of graphite rod, Nano Energy 2 (2013), 377-86.

[15] Papyex Flexible Graphite Technical Guide, https://www.mersen.com/uploads/tx_mersen/12-PAPYEX-flexible-graphiteMersen_07.pdf, Accessed October 2016.

[16] M.S. Dresselhaus, G. Dresselhaus, Intercalation compounds of graphite, Adv. Phys. 51 (2002), 1-186.

[17] D.D.L. Chung, Exfoliation of graphite, J. Mater. Sci. 22 (1987), 4190-8. 